\title{
Alteraciones puberales en adolescentes con leucemia en fase de vigilancia
}

\author{
Héctor Eliud Arriaga-Cázares', Mara Alejandra Cázares-Bellazetin, Luz María Sánchez-Sánchez, \\ Ana Laura Bahena-García ${ }^{1}$ y Gerardo del Carmen Palacios-Saucedo ${ }^{2}$ \\ ${ }^{1}$ Departamento de Pediatría; 'Departamento de Investigación en Salud. Centro Médico Nacional del Noreste, Unidad Médica de Alta Especialidad \\ No. 25, Instituto Mexicano del Seguro Social, Monterrey, N.L., México
}

\section{Resumen}

Objetivo: Evaluar qué factores se asocian a alteraciones en el desarrollo puberal en pacientes pediátricos con leucemia en fase de vigilancia. Método: Estudio de casos y controles, ambiespectivo, incluyendo pacientes de 8 a 14 años de edad con diagnóstico de leucemia linfoblástica aguda en fase de vigilancia. Se recolectaron datos demográficos, edad al diagnóstico, tipo de leucemia, riesgo de la leucemia, duración y tipo del tratamiento recibido, y tiempo de fase de vigilancia. Se valoró el desarrollo puberal con la escala de Tanner y se determinó la edad ósea. Se realizó ultrasonido pélvico para las mujeres, y valores de hormona luteinizante. Se utilizaron la prueba exacta de Fisher y la prueba U de Mann-Whitney. Resultados: Se incluyeron 25 pacientes pediátricos con diagnóstico de leucemia linfoblástica aguda de entre 8 y 14 años de edad, con una mediana de 8 años, de los cuales cuatro (16\%) presentaron alteraciones puberales: uno tenía retraso puberal y tres tenían pubertad adelantada. De las variables estudiadas, el antecedente de haber recibido radioterapia estuvo relacionado con alteraciones puberales $(p=0.03)$. Conclusiones: El antecedente de haber recibido radioterapia como parte del tratamiento en los pacientes con leucemia linfoblástica aguda es un factor de riesgo para desarrollar anomalías puberales.

PALABRAS CLAVE: Leucemia linfoblástica aguda. Alteraciones puberales. Radioterapia.

\begin{abstract}
Objective: To evaluate which factors are associated with alterations in pubertal development in pediatric patients with leukemia in the surveillance phase. Methods: A case-control study was carried out, including patients aged 8-14 years with diagnosis of acute lymphoblastic leukemia under surveillance. Demographic data were collected, age at diagnosis, type of leukemia, risk of leukemia, duration and type of treatment received, time of surveillance phase; and pubertal development was assessed by Tanner stage, bone age, pelvic ultrasound for women, and LH levels. Fisher's exact test and Mann-Whitney U-test were used. Results: Twenty-five pediatric patients with a diagnosis of acute lymphoblastic leukemia between 8 and 14 years of age with a median of 8 were included, only 4 (16\%) presented pubertal alterations, 1 had pubertal delay and 3 advanced puberty. The history of radiotherapy was related to pubertal alterations $(p=0.03)$. Conclusions: The antecedent of having received radiotherapy as part of the treatment in patients with acute lymphoblastic leukemia is a risk factor for developing pubertal abnormalities.
\end{abstract}

KEY WORDS: Acute lymphoblastic leukemia. Alterations in pubertal development. Radiotherapy.

\author{
Correspondencia: \\ Héctor Eliud Arriaga-Cázares \\ Abraham Lincoln, $\mathrm{s} / \mathrm{n}$ \\ Col. Nueva Morelos \\ C.P. 64180 , Monterrey, N.L., México \\ E-mail: hectoreliud@gmail.com
}

Fecha de recepción: 14-12-2016

Fecha de aceptación: 11-01-2017

DOI://dx.doi.org/10.24875/GMM.17003098
Gac Med Mex. 2017;153:903-906

Contents available at PubMed www.gacetamedicademexico.com 


\section{Introducción}

El número de niños sobrevivientes a la leucemia linfoblástica aguda se ha incrementado en los últimos años, asociado a la importante mejoría del tratamiento desde la introducción de la combinación de quimioterapias y las medidas preventivas para evitar la infiltración leucémica al sistema nervioso central, con un porcentaje reportado de remisión de la enfermedad de alrededor del $80 \%$. Sin embargo, con estos avances en el tratamiento y la mejoría de la sobrevida se han ido observando numerosas complicaciones de salud, aún no completamente descritas ${ }^{1,2}$.

Las alteraciones endocrinológicas en los pacientes con leucemia linfoblástica aguda se pueden presentar durante la enfermedad o secundarias al tratamiento incluso varios años después, y son dependientes de la edad de inicio de la enfermedad, el tratamiento, el sexo y el tiempo transcurrido desde la finalización del tratamiento. Entre estas complicaciones se presentan disfunción gonadal, infertilidad y trastornos del desarrollo puberal ${ }^{3,4}$.

Se ha reportado que la edad prepuberal al inicio del tratamiento no protege del daño gonadal; al contrario, los niños que recibieron radioterapia a temprana edad presentaron algún trastorno del crecimiento o del desarrollo de la pubertad, y hasta el momento no se ha identificado ningún marcador clínico, hormonal o ecográfico que sea suficiente para determinar si existe insuficiencia gonadal ${ }^{5,6}$.

El objetivo de este estudio es evaluar qué factores se asocian a alteraciones en el desarrollo puberal en pacientes pediátricos con leucemia en fase de vigilancia.

\section{Método}

Se realizó un estudio de casos y controles, observacional, longitudinal, ambiespectivo y analítico, en el que se incluyeron pacientes de 7 a 14 años y 11 meses de edad con diagnóstico de leucemia linfoblástica aguda en fase de vigilancia, atendidos en la Unidad Médica de Alta Especialidad (UMAE) No. 25 de enero de 2014 a agosto de 2015.

Se recolectaron datos demográficos, edad al diagnóstico, tipo de leucemia, riesgo de la leucemia, duración y tipo de tratamiento recibido, y tiempo de fase de vigilancia. Se valoró el desarrollo puberal mediante determinación del estadio Tanner, edad ósea, ultrasonido pélvico para las mujeres y valores de hormona luteinizante (LH) de los pacientes con leucemia linfoblástica aguda en fase de vigilancia atendidos en nuestro hospital (Tabla 1).

Se utilizaron las pruebas ji al cuadrado o exacta de Fisher para variables categóricas, y t de Student o U de Mann-Whitney para variables cuantitativas. Se consideró significancia estadística un valor de $p<0.05$, analizándose los resultados con el programa estadístico SPSS 15.0.

\section{Resultados}

Se incluyeron 25 pacientes pediátricos con diagnóstico de leucemia linfoblástica aguda de entre 8 y 14 años de edad, con una mediana de 10 años (8-15 años) con pubertad normal y 8 años (8-9 años) con pubertad alterada, en vigilancia por la consulta externa de UMAE No. 25, los cuales aceptaron participar en el estudio mediante consentimiento informado. De ellos, $10(40 \%)$ eran del sexo masculino y $15(60 \%)$ eran del sexo femenino, con resultados alterados de predomino en el sexo masculino, con $3(75 \%)$ pacientes, y $1(25 \%)$ del sexo femenino $(p=0.126)$; solo hubo uno con retraso puberal $(25 \%)$.

A todos los pacientes se les solicitó LH, edad ósea y ultrasonido pélvico en las niñas, y se les hizo una evaluación de Tanner y somatometría al momento de la consulta. Para su estudio, los pacientes fueron divididos según presentaran desarrollo puberal normal o anormal. Cuatro pacientes (16\%) presentaban desarrollo puberal alterado, de los cuales tres tenían desarrollo puberal adelantado y uno atrasado, mientras que 21 (84\%) tenían un desarrollo puberal normal y acorde a su edad.

La edad de diagnóstico de la leucemia linfoblástica aguda tuvo una mediana de 5 años (1-13 años) en los pacientes con desarrollo puberal normal y de 6 años (2-7 años) ( $p=0.716)$ en aquellos con desarrollo puberal anormal. Predominó el sexo masculino: $3(75 \%)$ con resultado alterado y 1 (25\%) del sexo femenino $(p=0.126)$. La leucemia linfoblástica aguda se clasificó según el riesgo: de los pacientes con pubertad normal, 15 fueron de riesgo habitual $(60 \%)$, uno fue de riesgo moderado (4\%) y nueve fueron de riesgo alto (36\%); de los pacientes con pubertad anormal, dos fueron de riesgo habitual (50\%), ninguno fue de riesgo moderado $(0 \%)$ y dos fueron de riesgo alto $(50 \%)(p=0.767)$.

En cuanto a la somatometría, el peso tuvo una mediana de $38 \mathrm{~kg}(23.5-75.5 \mathrm{~kg})$ en los pacientes con desarrollo puberal normal y de $38 \mathrm{~kg}(22.5-47 \mathrm{~kg})$ 
Tabla 1. Características clínicas, hematológicas, hormonas gonadotróficas y ultrasonido pélvico en 25 pacientes pediátricos con leucemia linfoblástica aguda en vigilancia en la UMAE No. 25

\begin{tabular}{|c|c|c|c|}
\hline & \multicolumn{2}{|c|}{ Desarrollo puberal } & \multirow[t]{2}{*}{ p } \\
\hline & $\begin{array}{l}\text { Normal } \\
(n=21)\end{array}$ & $\begin{array}{c}\text { Anormal } \\
(n=4)\end{array}$ & \\
\hline \multicolumn{4}{|l|}{ Sexo } \\
\hline Masculino & 7 & 3 & \multirow[t]{2}{*}{0.158} \\
\hline Femenino & 14 & 1 & \\
\hline Edad actual (años) & $10(8-15)$ & $8(8-9)$ & 0.788 \\
\hline Edad al diagnóstico (años) & $5(1-13)$ & $6(2-7)$ & 0.716 \\
\hline Edad ósea (años) & $11(7-16)$ & $9(8-11)$ & 0.821 \\
\hline Talla (cm) & $1.4(1.2-1.7)$ & $1.3(1.2-1.5)$ & 0.334 \\
\hline Peso (kg) & $38(23.5-75.5)$ & $38(22.5-47)$ & 0.593 \\
\hline Radioterapia & $2(50 \%)$ & $2(50 \%)$ & 0.030 \\
\hline \multicolumn{4}{|l|}{ Riesgo } \\
\hline Habitual & 13 & 2 & 0.651 \\
\hline Moderado & 1 & - & - \\
\hline Alto & 7 & 2 & 0.522 \\
\hline Recaída a gónadas & $0 / 7$ & $1 / 3$ & \\
\hline (varones) & & & 0.305 \\
\hline Recaídas a sistema nervioso central & 3 & 2 & 0.429 \\
\hline \multicolumn{4}{|l|}{ Tanner } \\
\hline 1 & 5 & - & \multirow[t]{5}{*}{0.747} \\
\hline II & 8 & 2 & \\
\hline III & 5 & 1 & \\
\hline IV & 2 & 1 & \\
\hline $\mathrm{VI}$ & 1 & - & \\
\hline Ultrasonido pélvico & 4 & - & 0.157 \\
\hline Normal & 10 & 1 & \\
\hline \multicolumn{4}{|l|}{ Anormal } \\
\hline Hormona luteinizante (mg/dl) & $1.49(0.06-8.16)$ & $0.88(0.2-2.99)$ & 0.796 \\
\hline
\end{tabular}

( $p=0.593$ ) en aquellos con desarrollo puberal anormal. La talla tuvo una mediana de $1.4 \mathrm{~m} \mathrm{(1.2-1.7} \mathrm{m)}$ en los pacientes con desarrollo puberal normal y de $1.3 \mathrm{~m}(1.2-1.5 \mathrm{~m})(\mathrm{p}=0.334)$ en aquellos con desarrollo puberal anormal.

En cuanto al antecedente de haber recibido radioterapia, en los pacientes sin alteración puberal se encontraron dos (50\%), y entre los pacientes que sí presentaron alteración puberal se aplicó radioterapia también a dos $(50 \%)(p=0.03)$.

En cuanto a la valoración de Tanner de los pacientes sin alteración puberal, se encontraron cinco $(23.80 \%)$ pacientes en estadio I, ocho $(38.09 \%)$ en estadio II, cinco $(23.80 \%)$ en estadio III, dos $(9.52 \%)$ en estadio IV y uno $(4.76 \%)$ en estadio V; de los pacientes que sí presentaron alteraciones puberales se encontraron dos $(50 \%)$ en estadio II, uno $(25 \%)$ en estadio III y uno (25\%) en estadio IV ( $p=0.747)$.

En cuanto a la recaída a gónadas, solo se estudió a los varones, ya que los testículos son uno de los órganos santuario que se toman en cuenta para el riesgo de la enfermedad, con alteración puberal $1 / 3(33 \%)$.

Dos pacientes (50\%) presentaron recaídas al sistema nervioso central de los pacientes con alteración puberal, y tres (14\%) pacientes de los que tenían desarrollo puberal normal $(p=0.126)$.

El ultrasonido pélvico en las niñas tuvo como resultado diez (26.6\%) anormales y cuatro (66.6\%) normales en las pacientes con desarrollo puberal normal, y uno $(6.6 \%)$ anormal de los pacientes con desarrollo puberal anormal $(p=0.157)$. 
Los resultados de las hormonas fueron los siguientes: hormona estimulante del folículo con una mediana de $3.8 \mathrm{mg} / \mathrm{dl}(0.8-20.2 \mathrm{mg} / \mathrm{dl})$ en los pacientes con desarrollo puberal normal y de $2.06 \mathrm{mg} / \mathrm{dl}$ $(0.26-6.91 \mathrm{mg} / \mathrm{dl})(p=0.440)$ en los pacientes con desarrollo puberal anormal; LH con una mediana de $1.49 \mathrm{mg} / \mathrm{dl}(0.06-8.16 \mathrm{mg} / \mathrm{dl})$ en los pacientes con desarrollo puberal normal y de $0.88 \mathrm{mg} / \mathrm{dl}(0.2-2.99 \mathrm{mg} / \mathrm{dl})$ $(p=0.796)$ en los pacientes con desarrollo puberal anormal; y estradiol con una mediana de $4.97 \mathrm{mg} / \mathrm{dl}$ (0-119 mg/dl) en los pacientes con desarrollo puberal normal y de $1.62 \mathrm{mg} / \mathrm{dl}(0.15-104 \mathrm{mg} / \mathrm{dl})(p=0.557)$ en los pacientes con desarrollo puberal anormal.

\section{Discusión}

En nuestro estudio reportamos anormalidades en la pubertad en cuatro pacientes (16\%), similar a lo reportado por Leung, et al. ${ }^{7}$, ya que el grupo, el diagnóstico y el tratamiento son homogéneos con nuestros pacientes. Encontramos una mayor frecuencia de anormalidades puberales en los varones (75\%): uno tuvo retraso puberal secundario a recaída a gónadas y los otros dos presentaron pubertad precoz. De las 15 mujeres, solo una tuvo pubertad precoz.

El antecedente de haber recibido radioterapia como parte del tratamiento es un factor de riesgo para desarrollar anomalías puberales. Esto concuerda con lo publicado previamente en otros artículos ${ }^{8,9}$.

Respecto a la edad del diagnóstico, el inicio del padecimiento no es un factor de riesgo para desarrollar alteraciones puberales y es igual a lo reportado la literatura ${ }^{10}$.
Aunque consideramos que este es un resultado de relevancia, es necesario el seguimiento en un mayor número de pacientes para mejorar nuestro estudio.

Suponemos que existe una alteración de la pubertad basándonos en la clínica y el estudio de gabinete. Consideramos adecuado realizar en un futuro pruebas dinámicas de función hormonal para mejorar el método diagnóstico.

A pesar de que en la mayoría de los pacientes la función gonadal parece estar preservada, consideramos necesario darle seguimiento $y$, de ser posible, valorar la función reproductiva.

\section{Bibliografía}

1. De Fine Licht S, Winther JF, Gudmundsdottir T, et al.; ALiCCS study group. Hospital contacts for endocrine disorders in Adult Life after Childhood Cancer in Scandinavia (ALiCCS): a population-based cohort study. Lancet. 2014;383:1981-9.

2. Van Den Berg H, Van Der Lelie J. Acute lymphoblastic leukaemia in puberty and adolescence. Ann Oncol. 2000;11:1375-9.

3. Landier W, Armenian S, Bhatia S. Late effects of childhood cancer and its treatment. Pediatr Clin North Am. 2015;62:275-300.

4. Howard SC, Pui $\mathrm{CH}$. Endocrine complications in pediatric patients with acute lymphoblastic leukemia. Blood Rev. 2002;16:225-43.

5. Ibáñez IM, Cruz-Martínez O, Parareda A, et al. Evaluación de la pubertad y la función gonadal en pacientes pediátricos supervivientes de un cáncer infantil. An Pediatr (Barc). 2007;66:331-9.

6. Alves CHB, Kuperman H, Dichtchekenian V, et al. Growth and puberty after treatment for acute lymphoblastic leukemia. Rev Hosp Clin Fac Med S Paulo. 2004;59:67-70.

7. Leung W, Hudson M, Zhu $Y$, et al. Late effects in survivors of infant leukemia. Leukemia. 2000;14:1185-90.

8. Labarta JI, De Arriba A, Sanz de Miguel P, et al. Secuelas endocrinológicas del tratamiento del cáncer en la infancia. Rev Esp Endocrinol Pediatr. 2011;(2).

9. Castro-Feijóo L, Pombo M. Pubertad precoz. An Pediatr Contin. 2006;4:79-87.

10. Pasqualini T, Rey R, Gottlieb S, et al. Secuelas endocrinológicas de tratamiento de las enfermedades oncológicas en la infancia y adolescencia. RAEM. 2009;46:24-38. 